\title{
COMPARAZIONE E METODO COMPARATO
}

\author{
di Giovanni Sartori
}

Sulla politica comparata e sul metodo che la sostiene scrivevo vent'anni fa (quasi) ${ }^{1}$. Ne scrivevo perché ne ero fautore. Ma l'esito, oggi, è stato di gran lunga inferiore alle aspettative. Come mai? E per via di una sopravvalutazione iniziale? O perché siamo andati inciampando nel corso del cammino? $\mathrm{O}$ per altre ragioni ancora? Rispondere a queste domande è come tirare un consuntivo. Dove stiamo? Dove ci conviene andare? $\mathrm{E}$ un dibattito da riaprire. Nel mio scritto, diciamo, originario, chiedevo nell'ordine 1) perché comparare, 2) cosa è comparabile, 3) come comparare. Gioverà ripetere le stesse domande nello stesso ordine.

\section{Perché comparare}

Perché comparare? Rispondevo: «La comparazione è un metodo di controllo delle nostre generalizzazioni... o leggi del tipo se .. allora... Dico un metodo di controllo perché, ovviamente, non è il solo» $(1971,8)$. Non ho cambiato parere; e nemmeno scoprivo, allora, la polvere. Ma con l'andare degli anni della funzione di controllo della comparazione si parla sempre meno, o anche per nulla. Przeworski arriva addirittura a sostenere che «la ricerca comparata non consiste di comparazioni ma di spiegazioni... Lo scopo generale della ricerca tra paesi è di capire» $(1987,35)^{2}$. Ma

1 La Politica Comparata: Premesse e Problemi (1971, 7-66). È lo scritto con il quale esordiva la «Rivista Italiana di Scienza Politica»; e dunque, nell'intento, un po' uno scritto «programma». Infatti vi notavo che la politica comparata «si colloca al centro della scienza politica contemporanea» (ivi, 63). Quanto allo stato della scienza politica in generale, vedi la mia valutazione, «Dove va la Scienza Politica», in Graziano (1986, 98-114).

${ }^{2}$ Che il comparare non richieda comparazione mi colpisce come un controsenso, una assurdità. E trovo sbrigativa, aggiungo, la tesi del volume di Przeworski e Teune $(1970,8)$ secondo la quale l'obiettivo della ricerca comparata è di «sostituire nomi di variabili ai nomi dei sistemi sociali».

RIVISTA ITALIANA DI SCIENZA POLITICA / a. XX, n. 3, dicembre 1990 
anche senza arrivare all'estremo di negare che la comparazione debba comparare, anche così la parola controllo è diventata desueta. Ragin scrive che il sapere comparato «fornisce la chiave del capire, spiegare e interpretare» $(1987,35)$; e Mayer «ridefinisce» (nel titolo, e in verità soltanto nel titolo) la politica comparata come un campo il cui intento è «costruire una teoria esplicativa empiricamente falsificabile» $(1989,12)$. Come si fa a dissentire? Tutti vogliamo capire, spiegare e interpretare. Ma perché comparando? A meno che non si voglia assurdamente sostenere che solo la comparazione «spiega», Przeworski, Ragin, Mayer e altri non ci spiegano quale sia la ragion d'essere della via comparata al sapere. Insomma, la domanda «perché comparare» resta inevasa. E se la politica (come anche la sociologia) comparata non ha più una propria funzione euristica, allora non merita occuparsene più di tanto.

$\mathrm{Ma}$ cosa è successo al «controllo»? Come mai non se ne parla più? Una prima spiegazione (su due) è del tutto accidentale e prettamente americana. Nella struttura didattica delle Università statunitensi la demarcazione di rito è tra politica americana e politica comparata, dove quest'ultima dizione sta per «altri paesi»e, in genere, uno solo. Pertanto chi studia il presidenzialismo USA è un americanista, mentre chi studia il solo presidenzialismo francese viene dichiarato comparatista. Sfido chiunque a spiegarmi come mai. Il fatto resta che il settore detto «politica comparata» è ormai densamente popolato da studiosi che non hanno mai paragonato nulla a nulla, che non hanno nessun interesse a comparare, e che sono non meno «parrocchiali» degli americanisti (che lo sono ad oltranza). Lo stato del campo è dunque che il grosso dei comparatisti USA nulla compara e, a fortiori, nulla controlla. Come se ne esce? La via di minor resistenza è di far finta di nulla, di dichiarare che si può comparare senza comparare, e di tacere sul controllo. Probabilmente Przeworski e gli altri si sono convinti che è proprio così. $\mathrm{Ma}$ così non è.

Dicevo che le spiegazioni sono due. La seconda è seria - di natura gnoseologica o epistemologica - e verte specificamente sul controllo. Comparare è sì mettere a confronto qualcosa con qualcosa d'altro; ma se si ambisce a controllare, allora il quesito a monte è: controllo di cosa? Di leggi? Se il termine viene inteso in senso fisicalista o comunque di stretta osservanza positivista, allora è lecito obiettare. Così Marradi $(1985,308)$ attacca il programma di «sottoporre a controllo la validità universale di una 'legge'» osservando che coloro che lo sottoscrivono non hanno «riflettuto seriamente sul significato del termine 'universale' (va- 
lido sempre e ovunque)». Se così, ha ragione. Ma il programma fisicalista è ormai abbandonato. Ormai le ambizioni nomotetiche delle scienze sociali non sono universali (nel senso onnispaziale e anche onnitemporale definito da Marradi) e investono leggi dichiarate law-like e messe di solito al condizionale sotto la forma se... allora. Definiamo «legge» così: «una generalizzazione provvista di potere esplicativo che coglie una regolarità» (Sartori 1984, 11). In tal caso direi che l'obiezione epistemologica cade ${ }^{3}$.

Preciso dunque così: paragonare serve a controllare - verificare o falsificare - se una generalizzazione (regolarità) tiene a fronte dei casi ai quali si applica ${ }^{4}$. S'intende che compariamo per tantissime ragioni. Per «situare», per imparare dalle esperienze altrui, per avere termini di paragone (chi non conosce altri paesi non conosce nemmeno il proprio), per meglio spiegare, e altre ragioni ancora. Ma la ragione che ci obbliga a comparare sul serio è il controllo. Prendiamo l'asserzione «le rivoluzioni sono causate da privazione relativa», oppure «i sistemi presidenziali sono sistemi di governo forti», oppure «i sistemi di mercato postulano la democrazia». Vero o falso? Per accertarlo bisogna guardarsi attorno, e cioè controllare comparando.

Resta il problema di come il comparare esplicito si rapporti al compare implicito e se, lungo questa via, si possa anche arrivare a un comparare inconscio. Va da sé che il controllo è tanto più «forte» e attendibile quanto più si fonda su comparazioni esplicite e sistematiche. Ma è chiaro che anche lo studio monografico di un americanista può essere implicitamente comparato. È tale se soddisfa la condizione che Zelditch $(1971,305)$ formula tecnicamente così: «la comparabilità postula un universale logico comune a tutte le unità di un universo di comparazione dato». Detto più discorsivamente, «implicitamente comparato» sta per parametri (prospettiva e proporzioni) ricavati da casi comparabili, e per l'impiego di categorie di analisi non-idiografiche, e cioè

\footnotetext{
${ }^{3}$ Marradi $(1985,310)$ incalza con l'ulteriore obiezione del «problema di Galton»: che «il parallelismo fra gli stati di due o più proprietà potrebbe essere dovuto ... a fenomeni di diffusione ... per via di conquista, imitazione ecc.» Confesso che il portato esiziale dell'obiezione di Galton mi sfugge. Certo, un «contagio» non avvertito ci espone a spiegazioni circolari; ma una volta avvertito può venire incorporato nella spiegazione genetica di una regolarità.

${ }^{4} \mathrm{Si}$ noterà che questa formulazione non ci impone di stabilire ex ante a che livello (globalizzante, intermedio, o strettamente «regionale») generalizzare. $\dot{\mathrm{E}}$ ragionevole o comunque prudente raccomandare, come fa Bendix $(1963,539)$, di sviluppare concetti e generalizzazioni a un livello intermedio tra ciò che è vero di tutte le società e ciò che è vero di una società. Ma la raccomandazione è prudenziale, non metodologica.
} 
derivate da una qualche teoria generale, da un qualche schema concettuale (framework) generalizzantes.

Fin qui tutto bene. Dobbiamo ancora fare i conti con la tesi che tutti siamo, per forza, volenti o nolenti, animali comparanti. La citazione d'obbligo è Durkheim $(1947,37)$, che scriveva: «La sociologia comparata non è un ramo della sociologia; è la sociologia stessa nella misura in cui... mira a rendere conto dei fatti». Lasswell $(1968,3)$ riprende il punto così: «l'approccio scientifico è inevitabilmente comparato». Più cautamente Almond (1970, 254): «Se [la scienza politica] è una scienza, va senza dire che è comparata nel suo approccio». Durkheim, Lasswell e Almond dicono male? No, se si legge con attenzione dicono che se il nostro approccio non è comparato, allora non è scientifico. Un grosso «se», dal quale si dovrebbe ricavare che tutti i «parrocchialisti» (in USA quasi tutti gli americanisti, e moltissimi comparatisti d'ufficio, tali per incauta definizione disciplinare) non fanno scienza, sono pseudopolitologi. A me questa conclusione va benissimo. Ma non va per niente bene a legioni di interessati che si troverebbero esposti a squalifica. La tesi era (è) che chi non compara non fa scienza; tesi che viene rigirata così: siccome facciamo scienza, vuol dire che tutti compariamo, anche se inconsciamente. La mossa è astuta, ma resta un imbroglio. Una cosa è ammettere la comparazione «implicita» (che è tale perché consapevole) e tutt'altra cosa avallare una comparazione «inconsapevole» e per ciò stesso presente e operante per definizione. Proprio no. Il comparare inconscio, o subconscio, non compara nulla e non surroga niente. Difatti al coperto del detto che siamo tutti «inevitabilmente comparativi» è fiorita una lussurreggiante selva oscura di ipotesi e generalizzazioni che sono patenti sciocchezze che andrebbero a picco in un attimo se sottoposte a controllo comparato.

${ }^{5}$ La prova di quanto la politica comparata sia davvero tale non è dunque fornita da quanto sia «caso singolo» e quanto «molti-casi», ma dalla differenza tra studi incomparabili (tali perché fondati su parametri e categorie ad hoc) e studi comparabili. Non è un prova o riprova difficile: basta guardare alle bibliografie di sostegno. Il grosso degli studi monografici che mi passano sott'occhio sull'America Latina, Giappone, ecc. cita solo scritti country-specific, bellamente e, anzi, spudoratamente ignorando la letteratura generale sui parlamenti, burocrazie, partiti, sistemi elettorali, e quant'altro l'autore osserva nel paese osservato. 


\section{Cosa è comparabile}

Se il comparare non ha una ragion d'essere distintiva, uno scopo specifico (oltre a quello generale di spiegare), alla stessa stregua si può sostenere che non ci sia, propriamente parlando, una metodologia della comparazione, un metodo comparato. A detta di Lijphart $(1971,682)$ la politica comparata è quel settore della scienza politica che si definisce «mediante una etichetta metodologica invece che sostantiva». Così anche Holt e Turner $(1970,5)$ : «nel suo significato ordinario, comparato si riferisce a un metodo di studio, non a un corpo sostantivo di sapere». Nel complesso la tesi, diciamo, metodologica ha retto all'assalto dei critici assai meglio che la tesi che comparare sia controllare. Difatti, volumi che si dichiarano e sono di metodo comparato continuano ad uscire. Ma vediamo meglio.

Intanto nessuno (o quasi) sostiene che il metodo comparato sia una entità a sé. Ovviamente è parte integrante del metodo delle scienze sociali in generale. Come ugualmente sarebbe assurdo sostenere che la logica della comparazione sia diversa dalla logica come tale. Ma l'albero del sapere cresce, e al tronco si aggiungono rami nuovi o più estesi. La logica di John Stuart Mill non rimpiazza Aristotele, ma certo aggiunge alla logica classica parti inedite. E, vedi caso, il metodo comparato sub specie di metodo logico discende in larga parte da Mill ${ }^{6}$. Per cominciare, mele e pere sono comparabili o no? Sfido chicchessia a rispondere in virtù di scienza infusa (tant'è vero che a tutt'oggi c'è chi risponde sì, chi risponde no, e chi non sa quali mele o pere pigliare). Dunque, sul punto la risposta è che il metodo comparato si giustifica e sviluppa come una specializzazione del metodo scientifico (scientifico-empirico o scientifico-logico) in generale.

Tutta diversa è la questione di come il metodo comparato si qualifichi e rapporti agli altri metodi di controllo che Lijphart indicava, specialmente il metodo sperimentale da un lato, e il metodo statistico dall'altro $(1971,683-85)$. Anche a questo proposito si può tornare a ripetere che tutto è comparato e che tutti - ivi inclusi lo sperimentatore e lo statistico - fanno paragoni. Vero,

${ }^{6} \mathrm{E}$ più precisamente dai suoi canoni sull'induzione, specialmente il metodo della concordanza e il metodo della differenza, che Cohen e Nagel riformulano così: i) «niente può essere causa di un fenomeno che non sussista come circostanza comune per tutte le manifestazioni del fenomeno» $(1934,255)$, e ii) «niente può essere causa di un fenomeno se il fenomeno non si dà quando si dà la sua supposta causa» (ivi, 259). Il terzo canone di Mill, il metodo della variazione concomitante, ne è una derivazione. 
ma triviale. Il punto resta che i tre metodi non sono equivalenti e nemmeno, spesso, opzionali ${ }^{7}$. Tengo ancor fermo, dunque, che «le scienze dell'uomo si avvalgono di quattro... tecniche di verifica. Seguendo un ordine di 'forza di controllo' decrescente... sono 1) il metodo sperimentale, 2) il metodo statistico, 3) il metodo comparato, 4) il metodo storico» (Sartori 1971, 8 e 8-13 passim). Il dubbio sopraggiunto è se sia proprio vero che il metodo statistico sia, quando possibile, sempre da preferire a quello nonstatistico. Alcuni autori (Frendreis 1983, 258; e soprattutto Ragin $1987,15-16)$ sostengono che il metodo statistico è sopravvalutato e che «il metodo comparato è superiore al metodo statistico per parecchi rispetti». Sia come sia, resta vero che di fronte a «molte variabili, piccolo $N \gg$ (Lijphart 1971, 686) la scelta è obbligata: il trattamento statistico è improponibile. $\mathrm{E}$ mi pare anche vero, come sostengono Ragin e Zaret $(1983,745)$, che «il metodo storico-qualitativo» non può essere considerato «una cruda approssimazione dei metodi statistici»: i rispettivi prodotti sono del tutto diversi. Ma veniamo alla sostanza.

Cosa è comparabile? Torniamo alle mele e pere. Sono comparabili o no? È l'esempio facile. Ma sassi e struzzi sono comparabili? Probabilmente i più risponderanno di no, per essere subito rimbeccati dall'obiezione: per dichiararli «incomparabili» li abbiamo pur dovuti, seppur per un solo attimo, comparare. Ricominciamo dal semplice. Mele e pere sono comparabili rispetto a talune proprietà - quelle in comune - e non comparabili rispetto ad altre. Così, mele e pere sono comparabili come frutta, come commestibili, come entità che crescono su alberi; ma non sono comparabili, per esempio, quanto a forma. In punto di principio, allora, la domanda è sempre da formulare così: comparabile (abbastanza simile) rispetto a quali proprietà o caratteristiche, e non comparabile (troppo dissimile) rispetto a quali altre proprietà o caratteristiche?

Da quanto sopra si ricava che il comparare assimila e diversifica nei limiti. Se due entità sono eguali in tutto, in tutte le loro caratteristiche, è come se fossero la stessa entità, e tutto finisce lì̀ ${ }^{8}$.

${ }^{7}$ Beninteso, si danno casi nei quali il controllo può essere tanto qualitativo-comparato quanto quantitativo-statistico. L'ipotesi sia che la coesione intra-partitica è una funzione diretta del grado di competizione inter-partitica, talché a minore competizione dovrebbe corrispondere un più elevato frazionismo interno dei partiti. In questo esempio la comparazione giova a raffinare l'ipotesi in modo da renderla correttamente controllabile mediante tecniche statistiche.

${ }^{8}$ Entità sta per qualsiasi unità: sistemi interi, «segmenti» sotto-sistemici (l'unità 
Viceversa, se due entità sono diverse in tutto, allora è inutile compararle, e di nuovo tutto finisce lì. Le comparazioni alle quali sensatamente attendiamo sono tra entità i cui attributi sono in parte condivisi (simili) e in parte non condivisi (e dunque dichiarati non paragonabili). Resta il quesito di Osgood $(1967,7)$ : quand'è che lo stesso è davvero lo stesso, e quand'è che il diverso è davvero diverso? $\grave{E}$ un quesito che turba molto una professione che ripete a mo' di cantilena che tutte le differenze sono di grado. Se così, il quesito di Osgood è davvero spinoso. Ma se ci ricordiamo ancora dell'analisi per genus et differentiam e non disdegnamo le classificazioni, allora il problema è subito risolto. Classificare è ordinare un universo in classi che sono mutuamente esclusive; pertanto classificare è di per sé stabilire cosa è eguale e cosa è diverso. S'intende che «eguale» è qui nozione relativa. Più precisamente, due oggetti che cadono nella stessa classe sono più simili tra loro - rispetto al criterio di assegnazione prescelto - che non agli oggetti che cadono in altre classi. Il che ci lascia, in principio, con gradi di similarità molto elastici. La regola di massima è che tanto minore è il numero delle classi, tanto maggiore sarà la variazione (dissimilarità) intra-classe. Viceversa, tanto maggiore è il numero delle classi, tanto minore la loro variazione interna. Se dividiamo gli Stati soltanto tra monarchia e repubblica otteniamo due classi troppo eterogenee per essere di una qualche utilità. $\mathrm{Ma}$ anche con classi minute i casi classificati assieme non sono mai del tutto eguali. Ed è competenza di chi classifica decidere quanto le sue classi debbano essere inclusive (poche e a larghe maglie) ovvero discriminanti (strette).

L'essenziale, ripeto, è che la domanda «cosa è comparabile?» venga sempre formulata così: comparabile in quale rispetto? In questa ottica pere e mele sono, in parecchi rispetti (proprietà), comparabili. Lo sono anche, ma meno, uomini e gorilla (per esempio sono entrambi animali eretti con mani prensili). Lo sono persino uomini e balene (in quanto mammiferi e animali che non possono respirare sott'acqua). S'intende che man mano che si passa dal primo al terzo accoppiamento le incomparabilità aumentano; ma fin qui non siamo in errore. Gli errori cominciano e si sviluppano attorno al «can-gatto»; un mio conio che illustro,

preferita da La Palombara, 1970), o anche, al limite, per una singola caratteristica. Le entità in questione possono essere cross-country, tra paesi, oppure essere interne, withincountry e anche multi-level. 
per rompere il ghiaccio, con una storiella immaginaria (ma non troppo).

\section{Il can-gatto}

Sempronio è ormai arrivato alla sua dissertazione, al $\mathrm{Ph}$. D. americano. Gli viene martellato in testa che la sua tesi dovrà essere originale e che dovrà ruotare attorno a una o più ipotesi. Sempronio si interessa ai gatti; ma come si fa a essere originali sui gatti? Pensa e ripensa, sceglie il can-gatto; e la sua ipotesi è che tutti i can-gatti emettono il suono miao, miao. Il relatore dice «interessante» e una fondazione elargisce 100.000 dollari per la ricerca. Tre anni dopo Sempronio ricompare, assai depresso. L'ipotesi, ammette, non è confermata: molti can-gatti emettono sì il suono miao, miao; ma molti no. Comunque, dice Sempronio, nel corso della ricerca mi è venuta in mente una ipotesi alternativa: che tutti i can-gatti emettono il suono bau, bau. Passano altri tre anni, vengono spesi altri 100.000 dollari, e ancora una volta l'ipotesi è smentita: è vero che molti can-gatti fanno bau, bau; ma molti no. Sempronio è disperato, e anche il suo relatore è imbarazzato, non sa più cosa suggerire. Alla fine lo consiglia di interpellare l'oracolo di Delfi. Sempronio arriva alla caverna di sera, e l'oracolo era stanco. Stanco anche di escogitare responsi sibillini. Lo ascolta, e mosso a pietà dice: amico mio, a te dirò il vero senza veli; la semplice verità è che il can-gatto non esiste.

Come nasce il can-gatto? Nasce da quattro fonti che si rinforzano l'una con l'altra: I) il parrocchialismo, II) il mal-classificare, III) il «gradismo» e, IV) la slargatura dei concetti.

Per parrocchialismo intendo gli studi di un solo paese in vacuo, che puramente e semplicemente ignorano le categorie di analisi poste da teorie generali e che quindi acciuffano a sproposito termini ritagliati su misura e, al tempo stesso, senza misura. Per esempio, Sundquist (1988) intitola e svolge un suo scritto in chiave di «governo di coalizione negli Stati Uniti». Ora, la dizione governo di coalizione si applica, attraverso il mondo, a sistemi parlamentari (non ai sistemi presidenziali di tipo americano) nei quali i governi dipendono dal parlamento e sono costituiti da alleanze di due o più partiti. Queste caratteristiche sono tutte cospicuamente assenti nella fattispecie che Sundquist denomina governo di coalizione. Nasce così un can-gatto (anzi, peggio, un 
can-pesce) che presto finirà nei computers e così impasticcerà tutta la teoria dei governi di coalizione correttamente detti.

Il can-pesce di Sundquist risulta da un puro e semplice sbaglio di nome, che è poi sbaglio di ignoranza parrocchiale. Il mal-classificare porta a mostri più «sofisticati», più raffinati. Si pensi alla etichetta «sistemi monopartitici», che viene o veniva riferita 1) ai cosiddetti one-party States degli Stati Uniti, al Giappone e, a volte, a Svezia, Norvegia e India, 2) al Messico, 3) ai paesi comunisti pre-1990. Ora i primi sono - nelle dizioni da me proposte - sistemi a partito predominante che appartengono a contesti competitivi (Sartori 1976, 192-201); il Messico è un sistema egemonico che permette una competizione «limitata» (ivi, 230-38); mentre i paesi comunisti sono, o sono stati, a partito unico nel senso proprio del termine, e cioè perché hanno vietato nel diritto e nel fatto qualsiasi altro partito (che non fosse, se c'era, un «partito burattino» consentito ed esibito a scopi di facciata). Dizioni a parte, in ogni caso si tratta di tre animali del tutto diversi. Riuniti in uno, otteniamo un can-gatto-tigre. Supponiamo che ci interessi scoprire la causa o cause che portano al monopartitismo. Huntington $(1970,11)$ ipotizza che «le origini sociali dei sistemi a partito unico sono da ascrivere... a una biforcazione», e cioè che «i sistemi ad un partito... tendono ad essere il prodotto o di una cumulazione di cleavages... oppure del prevalere di un allineamento di cleavage sopra altri». Giusto? Sbagliato? Non lo sapremo mai, poiché né questa né alcun altra ipotesi potrà mai passare il vaglio del nostro mostro a tre teste. La generalizzazione che magari regge per i gatti non varrà che in parte per $\mathrm{i}$ cani e quasi per nulla per le tigri. E l'errore qui è di classificazione - non solo di dizione - perché dicendo «partito unico» si ricorre a un criterio numerico di classificazione dei sistemi partitici; e un corretto classificare che discende da un solo fundamentum divisionis, da un solo criterio, necessariamente produce classi mutuamente esclusive, classi che non consentono che «uno» e «più di uno» vadano assieme. Dunque, soltanto una pseudoclasse può consentire a Giappone e Cina, Svezia e Messico, Stati Uniti e Albania, di essere assegnati a uno stesso contenitore.

Un ulteriore produttore di can-gatti è - dicevo - il «gradismo», che a sua volta risulta dall'abuso della massima secondo la quale tutte le differenze sono differenze di grado da disporre lungo un continuo di più-meno. Così, non ci sono sani o malati, ma più o meno sani-malati. Anche così, talvolta stiamo in piedi, talaltra siamo costretti a stare a letto; spesso viviamo in casa, ma ci 
capita anche di finire in ospedale. Qual è la demarcazione, il confine, tra i due stati? Nell'esempio la risposta è facile: abbiamo inventato il termometro a mercurio dal quale risulta che il punto di divisione è attorno ai 37 gradi. Ma nelle scienze sociali non c'è termometro e tantomeno esistono «gradi naturali» che stabiliscono le discontinuità del continuo. Qui i cut-off points, i punti di taglio, sono arbitrari, e cioè lasciati al nostro singolo decidere. Per esempio, Douglas Rae $(1971,93)$ rifiuta di concepire il bipartitismo come una classe o un tipo, e parla invece di una «competizione bipartitica» che definisce così: una situazione tale che «il primo partito ottiene in parlamento meno del $70 \%$ dei seggi, e i due primi partiti ottengono cumulativamente almeno il $90 \%$ dei seggi». Va bene? Come lo sa? Lo sa perché ha preso i sistemi classificati come bipartitici e ha tirato le somme. Anche così, perché 90 invece di, mettiamo, 88 per cento, e 70 invece di, mettiamo, 68 per cento? Non c'è ragione «ragionata» di fissare i tagli dove Rae li fissa, e non c'è ragione di non variarli. Variandoli si spostano i confini della fattispecie e per ciò stesso si ridefiniscono e ridistribuiscono i casi. Poco male? No, male, perché Rae si propone di accertare se esistano «leggi» sugli effetti dei sistemi elettorali; e variando i confini si fanno quadrare o non quadrare le leggi. Per l'appunto Rae è bravo di suo; ma il punto di principio, o di metodo, è che le eccezioni (e quindi smentite) alle regole sono sovente collocate nei paraggi dei tagli, cosicché basta variare di poco i cut-off points per cancellare le eccezioni o, viceversa, per scoprire smentite. In sostanza il «gradismo» crea animali a soffietto che possiamo manipolare così come il Governatore Gerry manipolava le circoscrizioni elettorali, dando luogo all'arte - il gerrymandering - che ne ha immortalato il nome.

Infine, e riassuntivamente, $\mathrm{i}$ can-gatti sono procreati a getto continuo dal concept stretching (Sartori 1970, 1034; 1971, 20), la slargatura dei concetti. Si prenda «costituzione». Se il termine è slargato al punto da significare qualsiasi forma di Stato, allora la generalizzazione «le costituzioni ostacolano la tirannide» ne esce abbondantemente smentita (mentre risulterebbe confermata se riferita alla accezione stretta o garantista del termine). Si prenda «pluralismo». Se tutte le società sono dichiarate, in una qualche accezione della parola, pluralistiche allora diventa indimostrabile che il pluralismo si lega e collega alla democrazia. Si prenda ancora «mobilitazione». Se la parola è estesa sino al punto da ricomprendere sia la partecipazione come attività volontaria, il mettersi in moto da sé, sia l'essere messi in moto per forza, l'etero-mozione 
(mobilitazione in senso proprio), allora abbiamo un can-tigre grazie al quale la generalizzazione (vera) che la partecipazione caratterizza la democrazia può essere (falsamente) dichiarata falsa. Un altro esempio di concetto slargato a tal punto da svanire in nulla è «ideologia». Nell'uso corrente la parola non ha più contrario; tutto è sempre ideologia per definizione, senza possibilità di prova contraria; e così falso-pensiero e ricerca della verità si confondono in una notte post-hegeliana nella quale tutta la zoologia è costituita da vacche grigie.

Dunque, con il can-gatto e derivati (can-tigre, can-pero, etc.) il controllo comparato viene vanificato. E più can-gatti produciamo, e tantomeno siamo in grado di generalizzare e verificare (o falsificare) su alcunché.

\section{Come comparare}

Supponiamo di avere ben fermato cosa è distintamente comparabile a cosa, in che rispetto. Rimane da stabilire quale sia la strategia comparativa da adottare. «A volte il comparatista sottolineerà le similarità, a volte le differenze. Guarderà alle differenze nei contesti che sono simili, o... cercherà analogie in sistemi differenti» (Dogan e Pelassy 1984, 127). Parallelamente si danno due approcci: scegliere sistemi più simili, oppure scegliere sistemi più contrastanti ${ }^{9}$. Nel primo caso il ricercatore mette assieme sistemi «vicini», e cioè simili in quante più caratteristiche possibili, il che gli consente di accantonare un alto numero di variabili sotto la clausola ceteris paribus, e cioè dichiarandole eguali. La presunzione è che i fattori comuni di paesi relativamente omogenei (le cosiddette «aree», come l'Europa Occidentale, l'America Latina etc.) sono irrilevanti al fine di spiegarne le differenze. L'ideale sarebbe qui di trovare entità simili in tutte le variabili salvo una, e cioè salvo quella che investe il fenomeno da spiegare. Per contro, nel secondo caso il ricercatore mette in relazione sistemi che differiscono quanto più possibile in tutto salvo che nel fenomeno da indagare. Nell'esempio di Przeworski e Teune (1970, 35), se i tassi di suicidio sono gli stessi tra Zuni, Svedesi e Russi (davvero

\footnotetext{
9 Quest'ultima, il most different systems design, è la strategia raccomandata da $\mathrm{Pr}$ zeworski e Teune (1970). Bene. Ma mi sfugge perché questa strategia debba necessariamente consistere di «analisi a livelli multipli» e osservare «comportamenti a un livello sottostante a quello di sistema» (ivi, 34).
} 
sistemi diversissimi), allora è chiaro che il fenomeno non può essere spiegato da fattori sistemici, fattori che sono pertanto da ignorare.

Fin qui non ci sono problemi. Ma il controllo comparato si riferisce a ipotesi generalizzanti, a «leggi» (quasi-leggi) rispetto alle quali il problema è posto dalle eccezioni. Più precisamente, quando e quante eccezioni uccidono una regola? Il problema resta apertissimo. E se non lo sistemiamo è tutto il comparare - tanto nel suo perché quanto nel suo come - che rischia il naufragio.

\section{Regole ed eccezioni}

Ripetiamo il quesito così: in che misura una regola può sopportare eccezioni? Il detto comune che le eccezioni confermano la regola non è ben detto; in verità sta per dire che la nozione di eccezione presuppone l'esistenza di regolarità; il che non ci aiuta granché.

Cominciamo dal fermare il punto che se una legge o regola è concepita «deterministicamente», allora una sola eccezione basta a infirmarla. Ma le leggi delle scienze sociali non sono da concepire deterministicamente, e cioè secondo la formula: data la causa è già dato l'effetto. No: data una causa l'effetto non è certo, non è noto e sicuro ex ante. Nelle scienze sociali, ho sempre sostenuto $(1979,52)$, non vale la determinazione causale ma invece la indeterminazione causale. Anche qui si danno cause; ma cause i cui effetti non sono «necessari» come lo sono nelle leggi naturali. Difatti siamo soliti dire che le nostre generalizzazioni, le nostre quasi-leggi, sono «probabilistiche». Sì, purché ci si intenda sul significato di probabilità. Se si tratta di probabilità nel senso statistico e matematico del termine, allora stiamo barando al gioco, perché le «leggi statistiche» sono, appunto, leggi statistiche; non leggi nel senso che ci interessa, e cioè generalizzazioni (regolarità) spieganti che comportano uno scire per causas, un capire fondato su cause. Che la durata media della vita sia, mettiamo, di 66 anni, o che la frequenza dei nati femmina sia superiore a quella dei nati maschi sono leggi o probabilità statistiche che accertano ma non spiegano. E dunque, quando diciamo che le leggi delle scienze sociali sono probabilistiche diciamo soltanto che sono «leggi di tendenza». Il che non risolve per nulla il problema che è davvero da risolvere: come trattare le eccezioni.

Visto che le leggi in questione non sono deterministiche, ne 
consegue che una o poche eccezioni le indeboliscono ma non bastano a ricusarle. Ciò premesso, supponiamo che le nostre leggi siano del tipo se allora dove il «se» è costituito non da condizioni sufficienti ma (ai fini dell'esercizio mentale in questione) da condizioni necessarie, le condizioni senza le quali nulla. In tal caso specificare le condizioni necessarie è specificare quando è che una legge si applica o meno; e aumentare il numero delle condizioni necessarie è restringerne l'ambito di applicazione. Ai nostri fini questo significa che le eccezioni sono una funzione delle condizioni necessarie nel senso che sono ridotte (eliminabili) aggiungendo ulteriori condizioni. La legge di Galileo sulla caduta dei gravi fallisce alla prova sperimentale se non viene circoscritta dalla condizione «caduta nel vuoto». Dunque un primo modo di affrontare il problema delle eccezioni è di ridurre l'ambito di applicazione di una legge precisandone meglio le condizioni. Un modo alternativo di procedere è poi di riformulare una legge in modo da incorporare le eccezioni nella sua stessa formulazione ${ }^{10}$. È solo dopo aver perseguito le due strategie ad esaurimento che una legge può essere salvata spiegandone le eccezioni con argomenti ad boc, di circostanza ${ }^{11}$. Ma in nessun caso una legge può essere dichiarata «deterministica... con le eccezioni menzionate» (Riker 1982, 761).

\section{Incommensurabilità e generalità}

Veniamo all'obiezione di fondo: è davvero possibile comparare? La paragonabilità di cui parliamo esiste davvero? È un po' come l'obiezione che si fa al tradurre da una lingua all'altra. I traduttori, suona la massima, sono «traditori». Sì, in un qualche senso lo sono. Quando io traduco me stesso mi riscrivo. Peraltro mi sono anche imbattuto in traduttori che migliorano il testo che traducono. $E$ anche se tradurre è in qualche misura tradire e in qualche misura riscrivere (riconcepire), Dante suona bene anche in inglese e Shakespeare anche in italiano. Insomma, il tradurre come creazione di autentica equivalenza è difficile ma non impos-

${ }^{10}$ Entrambe le strategie sono illustrate in riferimento alle «leggi» sulla influenza dei sistemi elettorali in Sartori (1984a, 16, 31, e passim).

11 In linea generale seguo Lakatos $(1970,116)$ per il quale una teoria $\mathrm{T}$ è falsificata, e quindi da respingere, «se e soltanto se un'altra teoria $T^{1}$ è stata proposta con le seguenti caratteristiche: 1) $\mathrm{T}^{1}$ possiede un contenuto empirico in eccesso a quello di $\mathrm{T}$... 2) $\mathrm{T}^{\prime}$ spiega il successo precedente di $\mathrm{T} \ldots$... 3) e parte del contenuto in eccesso di $\mathrm{T}^{\mathrm{t}}$ è provato». 
sibile. Ma al momento è in auge la tesi detta della «incommensurabilità dei concetti» che nega a qualsiasi effetto - ivi incluso il comparare - questa possibilità. E una tesi che trova il suo sostegno epistemologico in Feyerabend (1975) e che si formula, ad effetto della comparabilità, così: i nostri concetti sono a tal punto imbevuti di contesto, così fondamentalmente radicati nella loro rispettiva cultura, storia e località, da essere «incommensurabili» ${ }^{12}$. Ma siccome a me Feyerabend non fa nessuna impressione $^{13}$, nemmeno mi impressiona la «incommensurabilità» estrema, o estremizzata, da lui ispirata. Semmai è più vera la tesi contraria che i concetti sono generalizzazioni camuffate, contenitori mentali che amalgamano l'incessante fluire di percezioni sempre diverse e discrete. Ma mentre gli odierni sostenitori della incommensurabilità esagerano, e di parecchio, Dilthey e Rickert bene distinguevano, oltre un secolo $\mathrm{fa}$, tra sapere idiografico, tipico della storiografia, e sapere nomotetico, tipico della scienza naturale. Qui non si erige una prigione di incommensurabili chiusi in se stessi a mo' di monadi, ma si propone una alternativa tra studio «configurativo» e studio generalizzante; una alternativa che comporta profitti e perdite, ma che consente anche compensazioni reciproche. L'indagine monografica e lo studio del caso privilegiano la densità del capire individualizzante, di un Verstehen in profondità. Per contro l'indagine comparata sacrifica il capire-in-contesto - e del contesto - alla inclusività e proporzione generalizzante.

Al limite si deve operare una scelta. In pratica le due vie si possono in qualche modo e misura combinare ${ }^{14}$. Sta anche di fatto che il comparatista deve pur ricavare le sue informazioni da studi monografici e «configurativi» così come, viceversa, lo specialista di un solo paese che ignora l'apporto comparato si impoverisce (e peggio). Il punto è nitidamente formulato da Lipset

12 Per l'esattezza, in Feyerabend il contesto è «teorico», mentre per i sociologi e politologi che oggi cavalcano la tigre della incommensurabilità il contesto è soprattutto storico-culturale. Ma senza Feyerabend alle spalle la incommensurabilità dei suoi volgarizzatori avrebbe meno fiato.

${ }_{13}$ In Sartori (1984b) il contributo di Kotowski (pp. 403-451) analizza il concetto di rivoluzione, concetto che dovrebbe essere particolarmente sensibile al «teoreticismo» di Feyerabend: e invece no: il grosso delle caratteristiche di «rivoluzione» risulta condiviso tra marxisti e no. Vedi nel volume citato anche le mie critiche; ma specialmente l'analisi critica di Lane (1987).

${ }^{14}$ Lo spiega bene Bendix (introduzioni a Bendix 1964 e 1978). Tocqueville non è certo esclusivamente idiografico, e Max Weber gioca su entrambi i campi. Su entrambi vedi Smelser (1976, capp. 2 e 5). 
(1963, 9-10) così: «L'analista delle società deve scegliere tra un approccio primariamente storico o primariamente comparato... Ma non può ignorare l'altro. Senza l'esame di... paesi diversi è impossibile sapere in che misura un fattore dato abbia l'effetto che gli viene attribuito in un paese singolo».

Metodologicamente parlando, comunque, la scelta tra l'individualizzare e il generalizzare è una scelta che si pone e impone. Dopodiché interessa stabilire, sempre in chiave di metodo, come convertire o quantomeno collegare i due procedimenti. In un passo molto citato Verba ci presenta la loro convergenza come una sorta di vortice vizioso, di attorcigliamento che finisce in strangolamento (della politica comparata). Il passo merita di essere trascritto per intero. «Per essere comparatisti, ci viene detto, occorrono generalizzazioni o leggi ricomprendenti [covering laws] che si applicano a tutti i casi di un determinato tipo... Ma dove sono le leggi generali? Le generalizzazioni sbiadiscono quando guardiamo ai casi singoli. Aggiungiamo variabili intervenienti a variabili intervenienti. Ma siccome i casi sono pochi approdiamo a una spiegazione fatta su misura per ciascun caso. Il risultato finisce per essere di sapore idiografico o configurativo... Man mano che torniamo a introdurre più e più variabili nell'analisi al fine di arrivare a generalizzazioni che tengano per tutta una serie di sistemi politici, finiamo per introdurne tante da ricavarne casi unici» (Verba 1967, 113).

Quanto sopra è un resoconto di come ci siamo impasticciati e abbiamo fatto un pasticcio di tutto; ma certo non dà alcuna ricetta su come uscirne. «Dove sono le leggi generali?». Ovviamente (la domanda di Verba è retorica) non ci sono, né ci possono essere, visto che a tutt'oggi non ci siamo chiariti le idee su come formularle ${ }^{15}$ e visto, per soprammercato, che se anche una qualche legge generale fosse a portata di mano produrremmo subito un can-gatto atto ad affossarla. «Aggiungere variabile interveniente a variabile interveniente» è certo un modo controproducente di affrontare il problema. Da tempo (Sartori 1970, 1040-45; $1975,16-19 ; 1984 b, 44-46)$ ho proposto che un metodo atto a collegare universali e particolarità è di organizzare le nostre categorie lungo scale di astrazione governate dalla regola di trasformazione ( sia in direzione ascendente che discendente) per la quale la connotazione e la denotazione dei concetti sta in relazione inversa.

\footnotetext{
${ }^{15}$ Svolgo il punto, in riferimento alle leggi di Duverger sui sistemi elettorali, in 1984a, 11-16.
} 
Così, al fine di rendere un concetto più generale - incrementandone anche la capacità di viaggiare - ne dobbiamo ridurre le caratteristiche o proprietà. Viceversa, al fine di rendere un concetto più specifico - e quindi contestualmente più adeguato - ne dobbiamo accrescere le proprietà o caratteristiche. Non mi dilungo perché la scala di astrazione è già stata da me abbondantemente trattata altrove. Ma è come predicare ai sordi. In parte è perché per destreggiarsi nelle trasformazioni in oggetto occorre un minimo di addestramento logico (che invece sempre più brilla per la propria assenza). $E$ in parte è perché è infinitamente più facile invocare la incommensurabilità oppure lasciar fare per noi agli elaboratori ${ }^{16}$.

\section{Lo studio del caso}

Prima di concludere resta da fermare come la comparazione si rapporta allo studio del caso specificamente inteso come case study euristico, cruciale, o anche deviante ${ }^{17}$. In sostanza il caso viene espressamente scelto o perché si presta a generare ipotesi, o perché è «cruciale» ai fini della conferma-sconferma di una teoria. Quando è così è chiaro che analisi del caso e analisi comparata sono indagini complementari che si rinforzano l'una con l'altra. $\grave{E}$ altrettanto chiaro che gli studi del caso in questione debbono essere, per essere, implicitamente comparati. Il che non toglie che lo studio di un solo caso esula dal metodo comparato. Un solo caso, per quanto cruciale, non basta a confermare una generalizzazione (anche se ne aumenta la plausibilità) e nemmeno basta a sconfessarla (anche se la indebolisce). Ma tener ferma la distinzione tra case study e comparazione non stabilisce in alcun modo che quest'ultima sia, euristicamente, superiore al primo. Stabilisce soltanto che quando si arriva al controllo di insieme, allora serve la comparazione.

Serve, o dovrebbe servire? Già nella seconda metà degli anni

16 Una via spianata, tra l'altro, dallo spunto di Przeworski e Teune $(1970,12)$ che «in massima parte $\mathrm{i}$ problemi di unicità versus universalità sono ridefinibili come problemi di misurazione». Sarebbe bello; ma non è vero.

17 Il massimo propugnatore del case study è Eckstein (1975). Il problema è discusso anche da Lijphart $(1971,1975)$. I due autori divergono più nelle dizioni che nella sostanza. Così Lijphart insiste sul caso bypothesis generating, mentre Eckstein intende per «caso cruciale» un caso prescelto al fine di conferma-sconferma di una teoria. Inoltre il primo esplicita la nozione di «caso deviante», bene esemplificato da Lipset (1956), che studia appunto una «deviazione» dalla legge di ferro dell'oligarchia di Michels. 
sessanta Verba $(1967,113)$ si domandava: «come mai, con tanto movimento, c'è stato così poco movimento in avanti?». E rispondeva: «in parte la risposta sta nella asperità [toughness] del problema». Sì; ma solo in parte. La risposta intera è che, proprio perché la comparazione sul serio è tough, è difficile, proprio per questo una disciplina senza disciplina logica, metodologica e terminologica fa presto a naufragare. Nei mari chiusi si può navigare anche a vista. Ma nei mari aperti - i mari della comparazione - chi naviga a casaccio e senza bussola rischia a ogni momento di finire in secca. Ci viene addirittura detto (lo ricordavo all'inizio) che la politica comparata non consiste di comparazioni. Al tempo stesso nessuno più ci spiega (probabilmente perché la «nuova metodologia» ha perduto il logos e si riconosce quasi soltanto nella statistica) come trattare pere e mele e ancor meno il can-gatto. Il più recente Verba $(1985,28)$ si consola scrivendo che «le cose vanno in realtà meglio... ma... che il progresso non ci basta mai, e il progresso crea nuovi problemi e nuove frustrazioni»; e dunque che se «la politica comparata è, ed è stata, deludente... lo è in rapporto alle speranze... originarie, non... nei suoi risultati effettivi». No, non convengo.

Verba esagera nell'assoluzione. Ma nemmeno debbo esagerare io nella critica. Intanto, le mie riserve investono soprattutto lo sviluppo della scienza politica negli Stati Uniti e si rifanno alla assurda distinzione - richiamata all'inizio - tra americanisti e comparatisti, o meglio alla assurda interpretazione che ne è scaturita. In Italia i comparativisti multinazionali, per così dire, non sono legioni; ma quasi tutti i nostri politologi sono «implicitamente» comparativisti nel senso che i loro parametri sono comparati. In secondo luogo, e in generale, le mie critiche investono soprattutto la sotto-utilizzazione del metodo comparato. Non sostengo - sarebbe assurdo - che non si diano eccellenti lavori di ampia ed esplicita comparazione. Tale è oggi, per esempio, lo studio delle crisi democratiche o, viceversa, dei processi di democratizzazione ${ }^{18}$. Ma anche nei lavori di massimo spicco la formulazione delle ipotesi è, se c'è, timida, e il controllo comparato resta quasi sempre in sordina. Peccato. Con un minimo di consapevolezza e sicurezza metodologica in più, potremmo fare molto di più. I frutti sono appena dietro l'angolo. Perché non coglierli?

18 Vengono prontamente in mente i nomi di Giuseppe di Palma, Juan Linz, S.M. Lipset, Arendt Lijphart, Leonardo Morlino, Philippe Schmitter. E l'elenco è certo da completare. 


\section{Riferimenti bibliografici}

Almond, G. (1970), Political Theory and Political Science, ora in Political Development: Essays in Heuristic Theory, Boston, Little, Brown \& Co.

Bendix, R. (1963), Concepts and Generalizations in Comparative Sociological Studies, in «American Sociological Review», XXVIII, pp. 532-39.

- (1964), Nation Building and Citizenship, New York, Wiley.

- (1978), Kings or People, Berkeley, University of California Press, trad. it., Re o popolo, Milano, Feltrinelli, 1982.

Cohen, M.B. e E. Nagel, (1934), An Introduction to Logic and Scientific Method, London, Routledge and Kegan Paul.

Dogan, M. e D. Pelassy (1984), How to Compare Nations: Strategies in Comparative Politics, Chatham, Chatam House.

Durkheim, E. (1947), Les Regles de la Méthode Sociologique, Paris, Presses Universitaires de France.

Eckstein, H. (1975), Case Study and Theory in Political Science, in F.I. Greenstein e N.W. Polsby (a cura di), Handbook of Political Science, vol. 7, cap. 3. Reading, Addison-Wesley.

Fisichella, D. (a cura di), (1985), Metodo Scientifico e Ricerca Politica, Roma, La Nuova Italia Scientifica.

Feyerabend, P. (1975), Against Method, London, Verso.

Frendreis, J.P. (1983), Explanation of Variation and Detection of Covariation: The Purpose and Logic of Comparative Analysis, in «Comparative Political Studies», XVI, pp. 255-72.

Graziano, L. (a cura di) (1986), La Scienza Politica in Italia: Bilancio e Prospettive, Milano, Angeli.

Holt, R.T. e J.E. Turner (a cura di) (1970), The Methodology of Comparative Research, New York, Free Press.

Huntington, S.P. e C.H. More (a cura di) (1970), Authoritarian Politics in Modern Society, New York, Basic Books.

Kalleberg, A.L. (1966), The Logic of Comparison: A Methodological Note on the Comparative Study of Political Systems, in «World Politics», XIX, pp. 69-82.

Lakatos, I. (1970), Falsification and the Methodology of Scientific Research Programmes, in I. Lakatos e A. Musgrave (a cura di), Criticism and the Growth of Knowledge, London, Cambridge University Press.

Lane, J.E. (1987), Against Theoreticism, in «International Review of Sociology», III, pp. 149-185.

LaPalombara, J. (1970), Parsimony and Empiricism in Comparative Politics, in Holt e Turner (1970).

Lasswell, H. (1968), The Future of the Comparative Method, in «Comparative Politics», I, pp. 3-18.

Lijphart A. (1971), Comparative Politics and Comparative Method, in «American Political Science Review», LXV, pp. 682-693. 
- (1975), The Comparable-Cases Strategy in Comparative Research, in «Comparative Political Studies», VIII, pp. 158-177.

Lipset, S.M., M. Trow e J.S. Coleman (1956). Union Democracy, Glencoe, Free Press.

- (1963), The First New Nation: The United States in Historical and Comparative Perspective, New York, Norton.

Macridis, R.C. (1953), Research in Comparative Politics [Report of the Social Science Seminar on Comparative Politics], in «American Political Science Review» XXXXVII, pp. 641-75.

- (1955), The Study of Comparative Government, New York, Random House.

Marradi, A. (1985), Natura. Forme e Scopi della Comparazione: un Bilancio, in Fisichella (1985), pp. 293-321.

Mayer, L.C. (1989), Redefining Comparative Politics, Newbury Park, Sage

Osgood, C.E. (1967), On the Strategy of Cross-National Research into Subjective Culture, in «Social Science Information», VI.

Przeworski, A. e H. Teune (1970), The Logic of Comparative Social Inquiry, New York, Wiley.

Przeworski, A. (1987), Methods of Cross-National Research, 1970-83: An Overview, in M. Dierkes et. al. (a cura di), Comparative Policy Research: Learning from Experience, Aldershot, Gower.

Rae, D.W. (1971), The Political Consequences of Electoral Laws, New Haven, Yale University Press.

Ragin, C., e D. Zaret (1983), Theory and Method in Comparative Research, in «Social Forces», LXI, pp. 731-754.

Ragin, C.C. (1987), The Comparative Method: Moving Beyond Qualitative and Quantitative Strategies, Berkeley, University of California Press.

Riker, W.H. (1982), Two-Party System and Duverger's Law, in «American Political Science Review», LXXVI, pp. 753-66.

Sartori, G. (1970), Concept Misformation in Comparative Politics, in «American Political Science Review», LXIV, pp. 1033-53.

- (1971), La politica comparata: premesse e problemi, in «Rivista Italiana di Scienza Politica», I, pp. 7-66.

- (1976), Parties and Party Systems: A Framework for Analysis, New York, Cambridge University Press.

- (1979), La Politica: Logica e Metodo in Scienze Sociali, Milano, SugarCo.

- (1984a), Le 'Leggi' sull'Influenza dei Sistemi Elettorali, in «Rivista Italiana di Scienza Politica», XIV, pp. 3-40.

- (1984b), Guidelines for Concept Analysis, in Sartori, (a cura di), Social Science Concepts: A Systematic Analysis, Beverly Hills, Sage, pp. 15-85.

- (1987), Elementi di Teoria Politica, Bologna, Il Mulino.

Sartori, G., F.W. Riggs e H. Teune (1975), Tower of Babel: On the Definition and Analysis of Concepts in the Social Sciences, Pittsburgh, International Studies Association.

Smelser, N.J. (1966), Notes on the Methodology of Comparative Analysis 
of Economic Activity, in Transactions of the Sixth World Congress of Sociology, vol. 2, pp. 101-17, Evian, International Sociological Association.

- (1967), Sociology and the Other Social Sciences, in P.F. Lazarsfeld et al. (a cura di), The Uses of Sociology, New York, Basic Books.

- (1976), Comparative Methods in the Social Sciences, Englewood Cliffs, Prentice Hall.

Sundquist, J.L. (1988), Needed: A Political Theory for the New Era of Coalition Government in the United States, in «Political Science Quarterly», CIII, pp. 613-35.

Vallier, I. (a cura di), (1971), Comparative Methods in Sociology, Berkeley, University of California Press.

Verba, S. (1967), Some Dilemmas in Comparative Research, in «World Politics», XX, pp. 112-127.

- (1985), Comparative Politics: Where Have we Been, Where Are we Going?, in Wiarda (1985).

Wiarda, H.J. (a cura di) (1985), New Directions in Comparative Politics, Boulder, Westview Press.

Zelditch, M. (1971), Intelligible Comparisons, in Vallier (1971), pp. $267-$ 307. 